\title{
Role of IFT88 in icariin-regulated maintenance of the chondrocyte phenotype
}

\author{
WEI XIANG ${ }^{1}$, JIAMING ZHANG ${ }^{1}$, RUI WANG ${ }^{1}$, LIMEI WANG ${ }^{2}$, SHENGJIE WANG ${ }^{2}$, \\ YINGXING WU ${ }^{1}$, YONGHUI DONG ${ }^{1}$, FENGJING GUO ${ }^{1 *}$ and TAO XU ${ }^{2 *}$ \\ Departments of ${ }^{1}$ Orthopedics and ${ }^{2}$ Rehabilitation, Tongji Hospital, Tongji Medical College, \\ Huazhong University of Science and Technology, Wuhan, Hubei 430030, P.R. China
}

Received March 6, 2017; Accepted January 5, 2018

DOI: $10.3892 / \mathrm{mmr} .2018 .8486$

\begin{abstract}
Maintenance of the chondrocyte phenotype is crucial for cartilage repair during tissue engineering. Intraflagellar transport protein 88 (IFT88) is an essential component of primary cilia, shuttling signals along the axoneme. The hypothesis of the present study was that IFT88 could exert an important role in icariin-regulated maintenance of the chondrocyte phenotype. To this end, the effects of icariin on proliferation and differentiation of the chondrogenic cell line, ATDC5, were explored. Icariin-treated ATDC5 cells and primary chondrocytes expressed IFT88. Icariin has been demonstrated to aid in the maintenance of the articular cartilage phenotype in a rat model of post-traumatic osteoarthritis (PTOA). Icariin promoted chondrocyte proliferation and expression of the chondrogenesis marker genes, COL II and SOX9, increased ciliary assembly, and upregulated IFT88 expression in a concentration- and time-dependent manner. Icariin-treated PTOA rats secreted more cartilage matrix compared with the controls. Knockdown of IFT88 expression with siRNA reduced extracellular signal-regulated kinase (ERK) phosphorylation, and icariin upregulated IFT88 expression by promoting ERK phosphorylation. Thus, IFT88 serves a major role in icariin-mediated maintenance of the chondrocyte phenotype, promoting ciliogenesis and IFT88 expression by increasing ERK phosphorylation. Icariin may
\end{abstract}

Correspondence to: Professor Tao $\mathrm{Xu}$, Department of Rehabilitation, Tongji Hospital, Tongji Medical College, Huazhong University of Science and Technology, 1095 Jiefang Avenue, Wuhan, Hubei 430030, P.R. China

E-mail: xutao0101@yeah.net

Dr Fengjing Guo, Department of Orthopedics, Tongji Hospital, Tongji Medical College, Huazhong University of Science and Technology, 1095 Jiefang Avenue, Wuhan, Hubei 430030, P.R. China E-mail: fengjing_guo@126.com

${ }^{*}$ Contributed equally

Key words: intraflagellar transport protein 88, icariin, primary cilia, chondrocyte phenotype therefore be useful for maintenance of the cartilage phenotype during tissue engineering.

\section{Introduction}

Articular cartilage injury can trigger joint pain and dysfunction. Cartilage exhibits a poor capacity for self-repair. Cartilage degeneration after trauma or during disease may readily trigger osteoarthritis (1). Cartilage tissue engineering is considered to be a promising approach towards cartilage repair. Various scaffolds promoting cartilage generation may be utilized, and chondrocyte proliferation and differentiation can be guided by application of suitable biological stimuli. However, maintenance of the phenotype of regenerated cartilage, and long-term retention of normal function, remain challenging.

Icariin is a monomeric compound identified in extracts of Herba Epimedii; the protein exhibits a cardioprotective effect, may be used to treat osteoporosis, and has aphrodisiac qualities $(2,3)$. Icariin promoted B-cell lymphoma 2 (Bcl-2) gene expression and suppressed Bax gene expression, thus inhibiting ventricular remodeling and myocardial cell apoptosis (4). Icariin upregulated cyclic guanosine 3',5'-monophosphate (cGMP) levels and inhibited phosphodiesterase type 5, thus promoting nitric oxide (NO) release (a property of aphrodisiacs) (2). In the context of osteoporosis, icariin promoted osteogenic differentiation of rat bone marrow stromal cells (BMSCs) by activating the phosphoinositide 3-kinase (PI3K)-AKT-endothelial NO synthase (eNOS)-NO-cGMP-protein kinase G (PKG) signaling axis, triggering rapid phosphorylation of c-Jun N-terminal kinase (JNK), p38 kinase, and extracellular signal-regulated kinase (ERK) $(5,6)$. Li et al (7) demonstrated that an icariin-impregnated hydrogel scaffold efficiently promoted repair of supercritically sized osteochondral defects, and enhanced the integration of regenerated cartilage and subchondral bone in a rabbit model.

Primary cilia are non-motile microtubular organelles protruding from the surfaces of most eukaryotic cells. Cilia serve as 'antennae', detecting mechanical stress, and they engage in biochemical signal transduction from the extracellular environment (8). Intraflagellar transport 88 (IFT88) is a key ciliary protein that shuttles cargos along the ciliary axoneme $(9,10)$. In chondrocytes, primary cilia transmit mechanical stress and chemical signals (11-13). Primary 
cilia facilitate secretion of the cartilage matrix in association with the Golgi apparatus (14). Inhibition of IFT88 triggers ciliary disassembly and dysplasia of the epiphyseal plate (15). However, any role for IFT88 in maintenance of the chondrocyte phenotype remains poorly understood. In the present study, it was hypothesized that icariin might aid in maintenance of the chondrocyte phenotype by regulating IFT88 expression. Thus, the effects of icariin on IFT88 expression in progenitor chondrocyte ATDC5 cells and primary chondrocytes were examined.

\section{Materials and methods}

Cells and reagents. The progenitor chondrocytic cell line ATDC5 was purchased from the American Type Culture Collection (Manassas, VA, USA). Primary chondrocytes were obtained from the knee cartilage of 6 newborn (3 days old, 6-9 g) Sprague-Dawley (SD) rats of either sex (3 male and 3 female,). These SD rats were provided by the Experimental Animal Center (Tongji Hospital, Wuhan, China). All animals were maintained in the same housing conditions with free access to food and water (see below 'Animal experiments' section). Cells were cultured in Dulbecco's modified Eagle's medium/nutrient mixture F-12 (DMEM/F12) supplemented with 10\% (v/v) fetal bovine serum (FBS) and $100 \mathrm{U} / \mathrm{ml}$ penicillin/streptomycin. Icariin was purchased from the Cayman Chemical Company (Ann Arbor, MI, USA). The ERK inhibitor, PD0325901, was obtained from Selleckchem (Houston, TX, USA).

Cell viability assay. The effect of icariin on ATDC5 cell proliferation was evaluated with the aid of a CCK-8 kit (Wuhan Boster Biological Technology, Ltd., Wuhan, China). Cells (2,000/well) were seeded into 96-well plates and cultured in $100 \mu \mathrm{l}$ DMEM/F12 with $10 \%$ (v/v) FBS medium containing different concentrations of icariin (0.001-10 $\mu \mathrm{mol} / \mathrm{l})$. After $48 \mathrm{~h}, 10 \mu \mathrm{l}$ amounts of CCK-8 solution were added to the wells, followed by incubation at $37^{\circ} \mathrm{C}$ for $90 \mathrm{~min}$. Absorbance at $450 \mathrm{~nm}$ was measured using a microplate reader.

Histochemical and immunohistochemical assays. Chondrocytes (obtained from the aforementioned newborn rats) and the knee joints of 8-week old SD rats described below, were subjected to histochemical staining. Cells were fixed in $4 \%$ (v/v) paraformaldehyde for $15 \mathrm{~min}$, and subsequently stained with $0.5 \%(\mathrm{v} / \mathrm{v})$ toluidine blue. The knee joints were fixed in $4 \%(\mathrm{v} / \mathrm{v})$ paraformaldehyde for 2 days, decalcified for 4 weeks in $10 \%$ (w/v) EDTA, embedded in paraffin, and stained with Safranin O-Fast Green and toluidine blue, following standard protocols. All immunohistochemical techniques followed were as described previously (16). Primary antibodies were obtained from Proteintech (Wuhan Sanying; Wuhan, China). Aggrecan expression in tissue sections was evaluated microscopically and its color intensity of staining was quantified by use of Image Pro Plus 6.0 (Media Cybernetics, Inc., Rockville, MD, USA) (17). The osteoarthritis assessment system of O'Driscoll was used to evaluate the severity of cartilage degeneration (18).

Immunofluorescence assay. Chondrocytes at appropriate densities were inoculated on to coverslips. Using standard immunofluorescence methods, the primary cilia were stained with anti-acetylated $\alpha$-tubulin antibody (cat. no. T7451; 1:300 dilution; Sigma-Aldrich; Merck KGaA, Darmstadt, Germany) or double-stained with that antibody together with an antibody against IFT88 (cat. no. AP11138b; 1:50 dilution; Abgent Inc., San Diego, CA, USA). CY3-conjugated goat anti-mouse, fluorescein isothiocyanate (FITC)-conjugated goat anti-rabbit immunoglobulin G (IgG) (cat. no. BA1031, 1:200 dilution; and cat. no. BA1105, 1:100 dilution; respectively; both from Wuhan Boster Biological Technology, Ltd.) served as secondary antibodies, and nuclei were stained with DAPI $(1 \mu \mathrm{g} / \mu \mathrm{l})$. Images were captured with a camera fitted to a fluorescence microscope.

Western blotting. Total cellular lysates were prepared with the aid of RIPA buffer. Samples of protein $(20 \mu \mathrm{g})$ were loaded into lanes, separated on sodium dodecyl sulfate (SDS)-polyacrylamide $10 \%$ gels, and transferred to polyvinylidene difluoride (PVDF) membranes. The PVDF membranes were incubated with antibodies against IFT88 (cat. no. AP11138b; 1:200 dilution; Abgent Inc.), ERK and phosphorylated ERK (cat. nos. 9102 and 4370, respectively; both 1:1,000; both from Cell Signaling Technology, Inc., Danvers, MA, USA), and GAPDH (cat. no. BM3876; 1:400 dilution; Wuhan Boster Biological Technology, Ltd.); and subsequently with horseradish peroxidase (HRP)-labelled goat anti-mouse or goat anti-rabbit secondary antibody (cat. nos. BA1050 and BA1054, respectively; both 1:2,000 dilution, both from Wuhan Boster Biological Technology, Ltd.). Protein bands were detected using a Bio-Rad enhanced chemiluminescence system (Bio-Rad, Philadelphia, PA, USA).

siRNA transfection. ATDC5 progenitor chondrocytic cells were transfected with $100 \mathrm{nM}$ siRNA targeting IFT88 or a negative control siRNA (Guangzhou RiboBio Co., Ltd., Guangzhou, China), using a standard protocol. Knockdown efficiency was evaluated by western blotting.

Quantitative polymerase chain reaction ( $q P C R$ ). Expression levels of genes affecting the chondrocyte phenotype (COL-II, SOX9, and IFT88) were measured by qPCR. Total RNA was extracted with TRIzol and cDNAs were synthesized from $2 \mu \mathrm{g}$ amounts of total RNA using a Toyobo cDNA synthesis kit (Toyobo, Co., Ltd., Osaka, Japan), according to the manufacturer's protocol. Each PCR tube contained $1 \mu \mathrm{l}$ cDNA, $1 \mu \mathrm{l}$ primers, $10 \mu \mathrm{l}$ SYBR-Green DNA polymerase (Toyobo, Co., Ltd.), and $8 \mu \mathrm{l}$ RNAse-free water. The primers sequences are listed in Table I.

Animal experiments. The present study was approved by the Ethics Committee of Tongji Hospital (Wuhan, China). A total of 24 male SD rats ( 8 weeks old, 180-220 g) were divided into four groups (control, treadmill, icariin, and icariin + treadmill). Animals were purchased from the Experimental Animal Center, Tongji Hospital, (Wuhan, China). All these rats were maintained with free access to food and water, at a constant room temperature of $23 \pm 1^{\circ} \mathrm{C}$ with a $12 \mathrm{~h} \mathrm{light/dark} \mathrm{cycle,}$ $50-70 \%$ humidity and $0.03 \% \mathrm{CO}_{2}$. Full-thickness cartilage defects were created, and early treadmill exercise was used 
Table I. Primer sequences.

\begin{tabular}{llcl}
\hline Gene (rat) & \multicolumn{1}{c}{ Primer sequences (5'-3') } & Gene (mouse) & \multicolumn{1}{c}{ Primer sequences (5'-3') } \\
\hline COLII & F: TCCTCCGTCTACTGTCCA & COLII & F: GCTCCCAACACCGCTAACG \\
& R: ACTTACCGGTGTGTTTCG & & R: GCCGCTTCGTCCAGGTAGG \\
SOX9 & F: TCGGGGCTCTACTCCACCT & SOX9 & F: GAGCCGGATCTGAAGAGGGA \\
& R: TCTGTCACCATTGCTCTTC & & R: GCTTGACGTGTGGCTTGTTC \\
IFT88 & F: ACCAGGCTGTAGACACATT & IFT88 & F: TGGCCAACGACCTGGAGATTAACA \\
& R: TTCTCGTAGTCACCATTTG & & R: ATAGCTGCTGGCTTGGGCAAATTC \\
GAPDH & F: CTGCTCCTCCCTGTTCTA & GAPDH & F: GCCTTCCGTGTTCCTACCC \\
& R: CAATGTCCACTTTGTCAC & & R: GCCCTCAGATGCCTGCTTC
\end{tabular}

R, reverse; F, forward; IFT88, intraflagellar transport protein 88 .

A
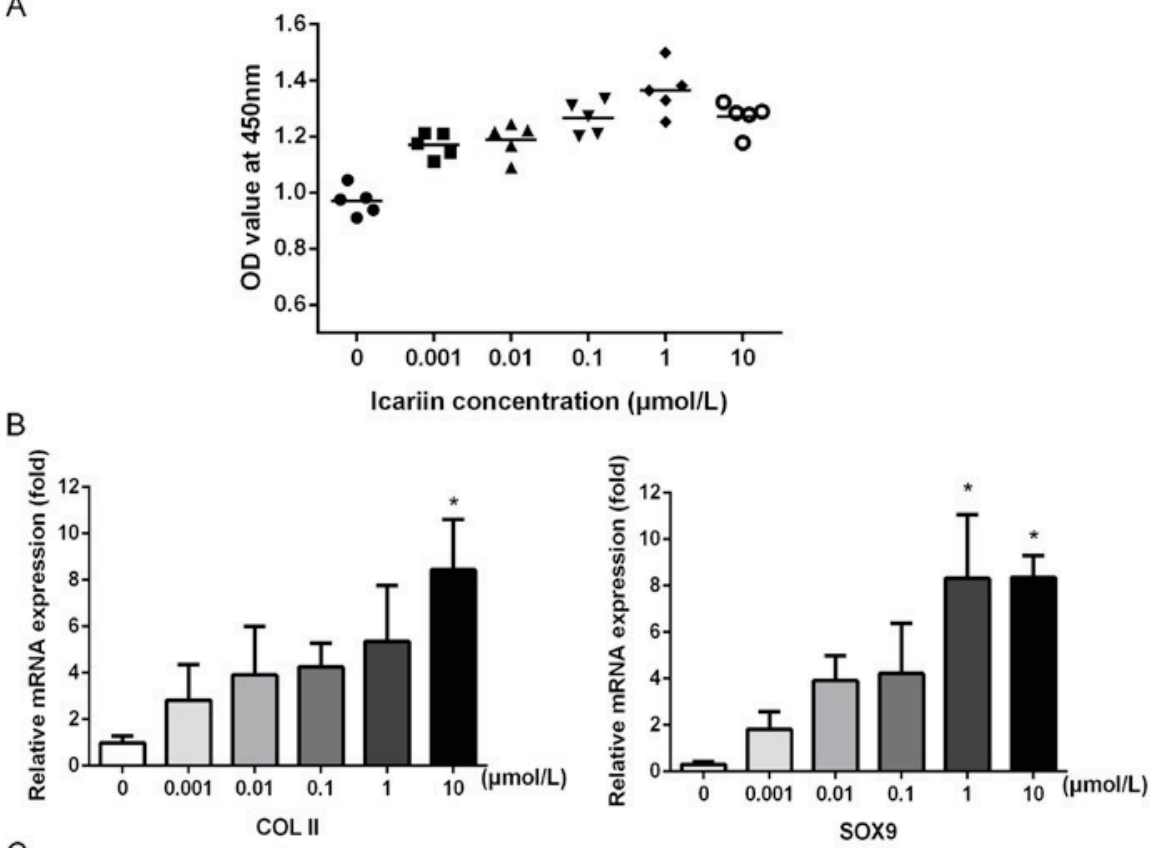

C

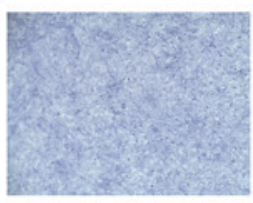

Control

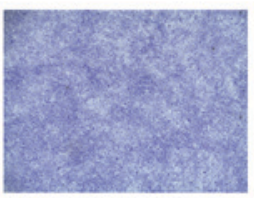

Icariin

$\times 100$

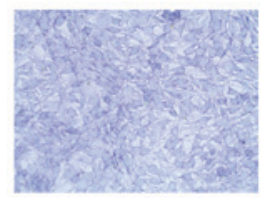

Control

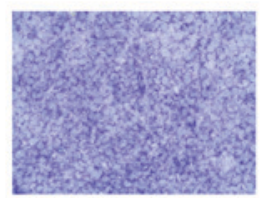

Icariin

$\times 400$

Figure 1. Icariin regulates ATDC5 cell proliferation and differentiation. (A) Icariin promoted ATDC5 cell proliferation in a concentration-dependent manner, peaking at $1 \mu \mathrm{mol} / 1$. (B) Relative mRNA expression levels detected by real time-quantitative polymerase chain reaction. Icariin enhanced the expression of the cartilage phenotype-associated genes, COL-II and SOX9, peaking at $10 \mu \mathrm{mol} / 1$. (C) Toluidine blue staining of the ECM (magnification x100 or x400). Icariin promoted ECM secretion. "P<0.05 compared with $0 \mu \mathrm{mol} / 1$ icariin. ECM, extracellular matrix.

to accelerate the development of post-traumatic osteoarthritis (PTOA). All rats underwent 1 week of treadmill training prior to surgery. Full-thickness cartilage defects were created by drilling a 1-mm-diameter hole through the middle of the femoral trochlea of the right knee, as described in a previous study (19). Rats in the treadmill and icariin + treadmill groups commenced running exercises $(15 \mathrm{~m} / \mathrm{min}, 30 \mathrm{~min} /$ day $) 1$ week after surgery (19), and were gavage-fed with icariin $(25 \mathrm{mg} / \mathrm{kg})$ or saline daily (20). After 6 weeks, the rats were sacrificed and knee samples were subjected to histomorphological and immunohistochemical assessment.

Statistical analysis. All data are reported as the mean \pm standard deviation, and means with $95 \%$ confidence intervals were calculated. Student's t-test or one-way analysis of variance was used to assess the significance of between-group 
A
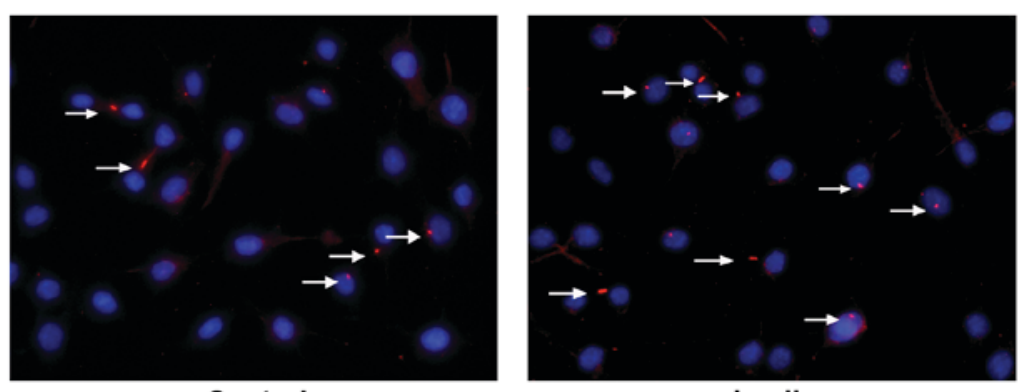

B
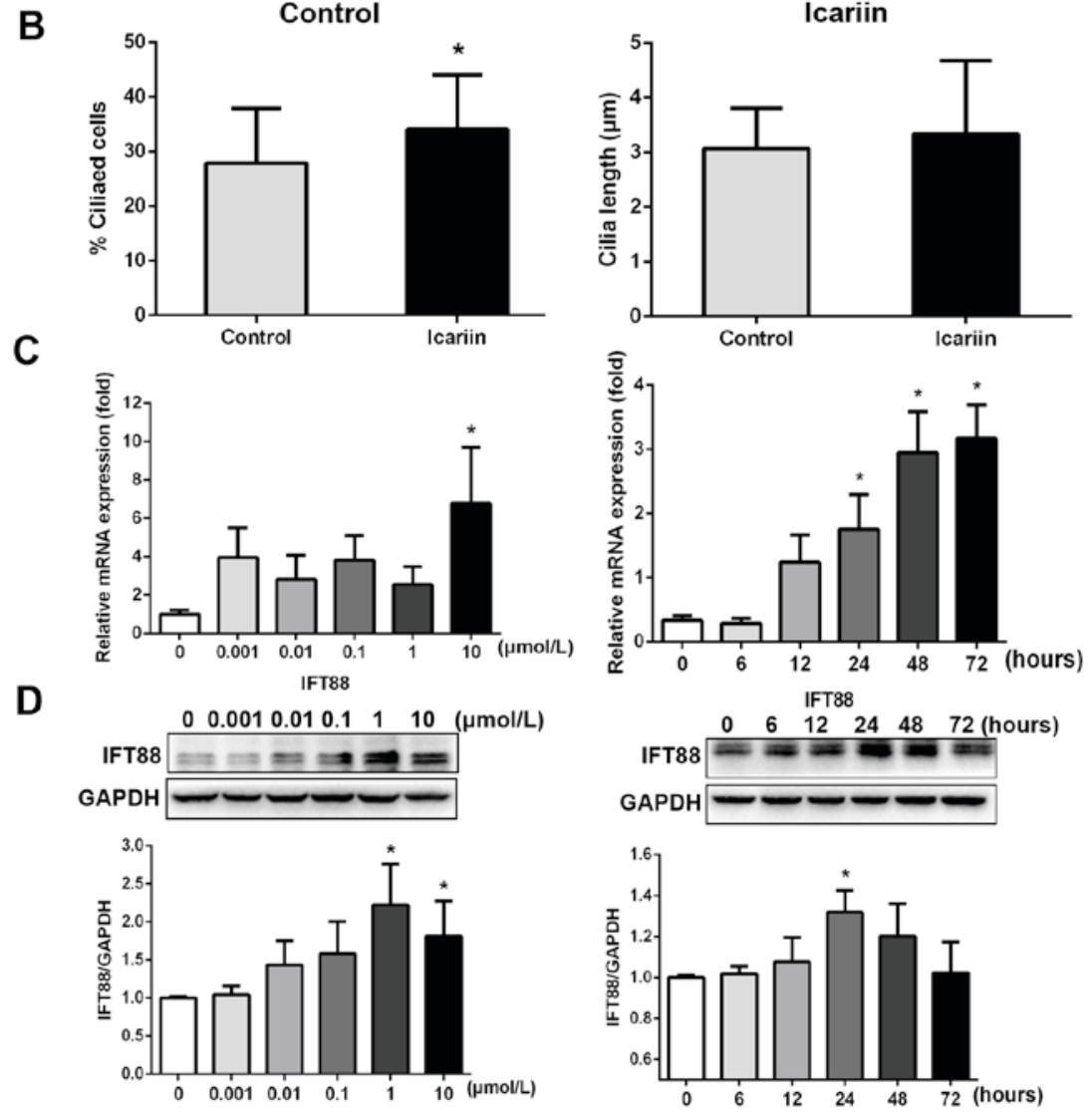

Figure 2. Icariin promotes ciliary assembly and IFT88 expression. (A) Primary cilia were stained for acetylated- $\alpha$-tubulin (red coloration, denoted by the white arrows; magnification, x200). (B) The histograms reveal that, compared with the control group, $10 \mu \mathrm{mol} / \mathrm{l}$ icariin increased primary ciliary assembly (Control group, $27.91 \pm 9.95 \%$ cf. Icariin, $34.06 \% \pm 10.06$; ${ }^{*} \mathrm{P}<0.05$ ), and icariin moderately increased the ciliary length from $3.07 \pm 0.74$ to $3.34 \pm 1.34 \mu \mathrm{m}$. (C) Icariin increased IFT88 gene expression, peaking at $10 \mu \mathrm{mol} / 1$ ("P<0.05 cf. $0 \mu \mathrm{mol} / 1$ icariin). (D) Icariin upregulated production of the ciliary protein, IFT88, in a concentration- and time-dependent manner ( $\mathrm{P}<0.05 \mathrm{cf} .0 \mu \mathrm{mol} / \mathrm{l}$ icariin, or treatment at $0 \mathrm{~h}$ ). IFT 88 , intraflagellar transport protein 88 .

differences. $\mathrm{P}<0.05$ was considered to indicate a statistically significant difference.

\section{Results}

Icariin affects the proliferation and differentiation of ATDC5 chondrocytic cells. Icariin at different concentrations, added to growing cells, promoted ATDC5 proliferation in a concentration-dependent manner, peaking at $1 \mu \mathrm{mol} / 1$ (Fig. 1A). Furthermore, icariin promoted the expression of the chondrocyte phenotype-associated genes, COL-II and SOX9, in a concentration-dependent manner, peaking at $10 \mu \mathrm{mol} / \mathrm{l}$ (Fig. 1B). Following stimulation with $10 \mu \mathrm{mol} / 1$ icariin for 3 days, toluidine blue staining confirmed that icariin significantly promoted secretion of cartilage extracellular matrix (ECM) (Fig. 1C).
Icariin promotes ciliary assembly in, and IFT88 expression by, ATDC5 cells. The primary cilia regulate numerous cellular activities, particularly the balance between proliferation and differentiation $(21,22)$. ATDC5 cells treated with $10 \mu \mathrm{mol} / 1$ icariin were analysed in terms of primary cilia production (Fig. 2A and B). Icariin-treated cells expressed a higher percentage of primary cilia $(34.06 \pm 10.06 \%$ of cells) compared with the control group $(27.91 \pm 9.95 \%)$. The average ciliary length of icariin-treated cells $(3.34 \pm 1.34 \mu \mathrm{m})$ was slightly greater compared with that of the control cells $(3.07 \pm 0.74 \mu \mathrm{m})$. IFT88 was expressed at both the gene and the protein level. RT-qPCR revealed that icariin enhanced the expression of mRNA encoding IFT88 in a concentration-dependent manner, peaking at $10 \mu \mathrm{mol} / 1$ (Fig. 2C). Icariin promoted the protein expression of IFT88 in a concentration- and a time-dependent manner, peaking at 1 or $10 \mu \mathrm{mol} / 1$. When exposed to $10 \mu \mathrm{mol} / 1$ 
A

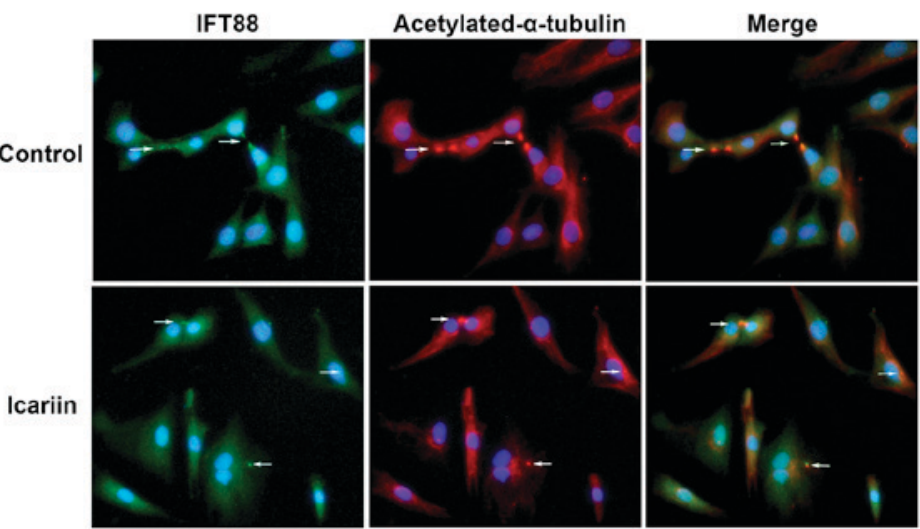

B

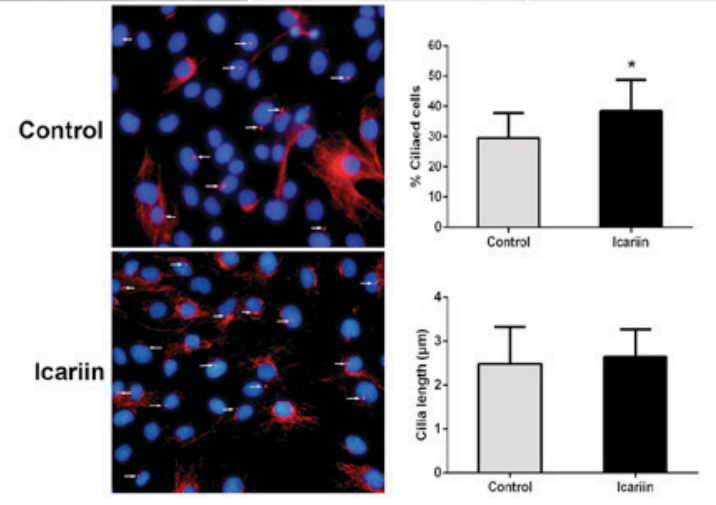

C

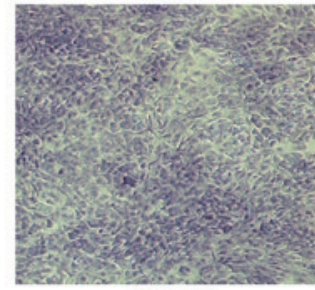

Control

D
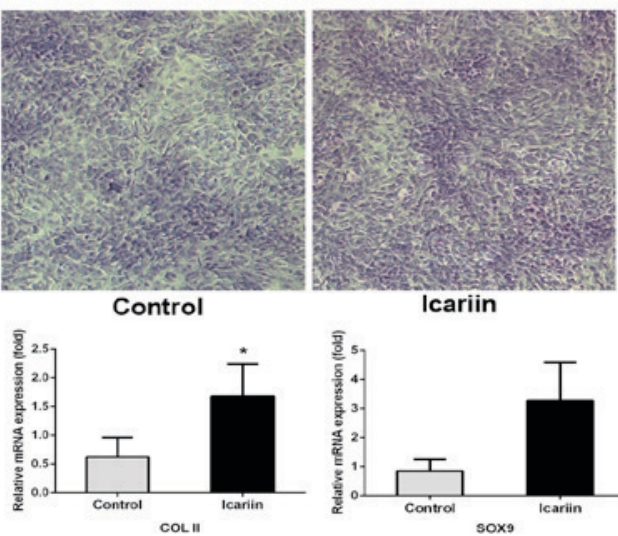

Icariin

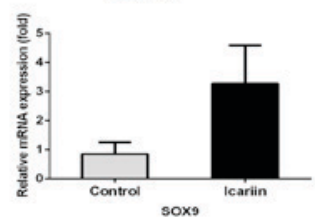

Figure 3. Icariin enhances primary ciliary assembly and maintains the phenotype of the primary chondrocytes. (A) Primary cilia were stained for acetylated- $\alpha$-tubulin (red). Functional IFT88 (green) was widely dispersed in the cytoplasm, although it was principally centralized along the ciliary axonemes, which also contained acetylated $\alpha$-tubulin (denoted by the white arrows; magnification, x200). (B) Icariin increased the proportion of primary chondrocytes with cilia, from $29.54 \pm 8.24$ to $38.48 \pm 10.36 \%$, and led to a slight elongation of the cilia from $2.48 \pm 0.85$ to $2.65 \pm 0.63 \mu \mathrm{m}$ ("P<0.05). (C) Toluidine blue staining revealed that icariin promoted cartilage matrix secretion by primary chondrocytes. (D) Expression of phenotype-associated genes in primary chondrocytes $($ P $<0.05)$. IFT88, intraflagellar transport protein 88.

icariin for different times, the protein expression of IFT88 peaked at $24 \mathrm{~h}$ (Fig. 2D).

Icariin facilitates ciliary assembly in primary chondrocytes and assists in maintenance of an appropriate phenotype. Primary chondrocytes were treated with icariin $(10 \mu \mathrm{mol} / \mathrm{l})$ for $24 \mathrm{~h}$, and the levels of primary cilia and IFT88 were measured. IFT88 protein was detected in the cytoplasm, although it was principally centralized along the axonemes of primary cilia (Fig. 3A). Icariin-treated primary chondrocytes exhibited enhanced ciliary assembly $(38.48 \pm 10.36 \%)$ compared with that of the control cells $(29.54 \pm 8.24 \%)$, and a slight increase in ciliary length was revealed (from $2.48 \pm 0.85$ to $2.65 \pm 0.63 \mu \mathrm{m}$; Fig. 3B). Toluidine blue staining confirmed that icariin-treated primary chondrocytes secreted more cartilage matrix and expressed higher levels of the COL-II and SOX9 genes than did the control cells (Fig. 3C and D).

Icariin attenuates cartilage degeneration in the PTOA rat model. Toluidine blue and Safranin O-Fast Green staining revealed that the treadmill and treadmill + icariin groups exhibited reductions in cartilage thickness in weight-bearing areas, whereas the treadmill group lost more of the superficial cartilage proteoglycans than did the treadmill + icariin group. Icariin-treated and control rats had thicker cartilages that were rich in proteoglycans (Fig. 4A). Subsequently, the expression of aggrecan (a major component of the cartilage ECM that allows cartilage to resist compression) was explored (23). Aggrecan 
A

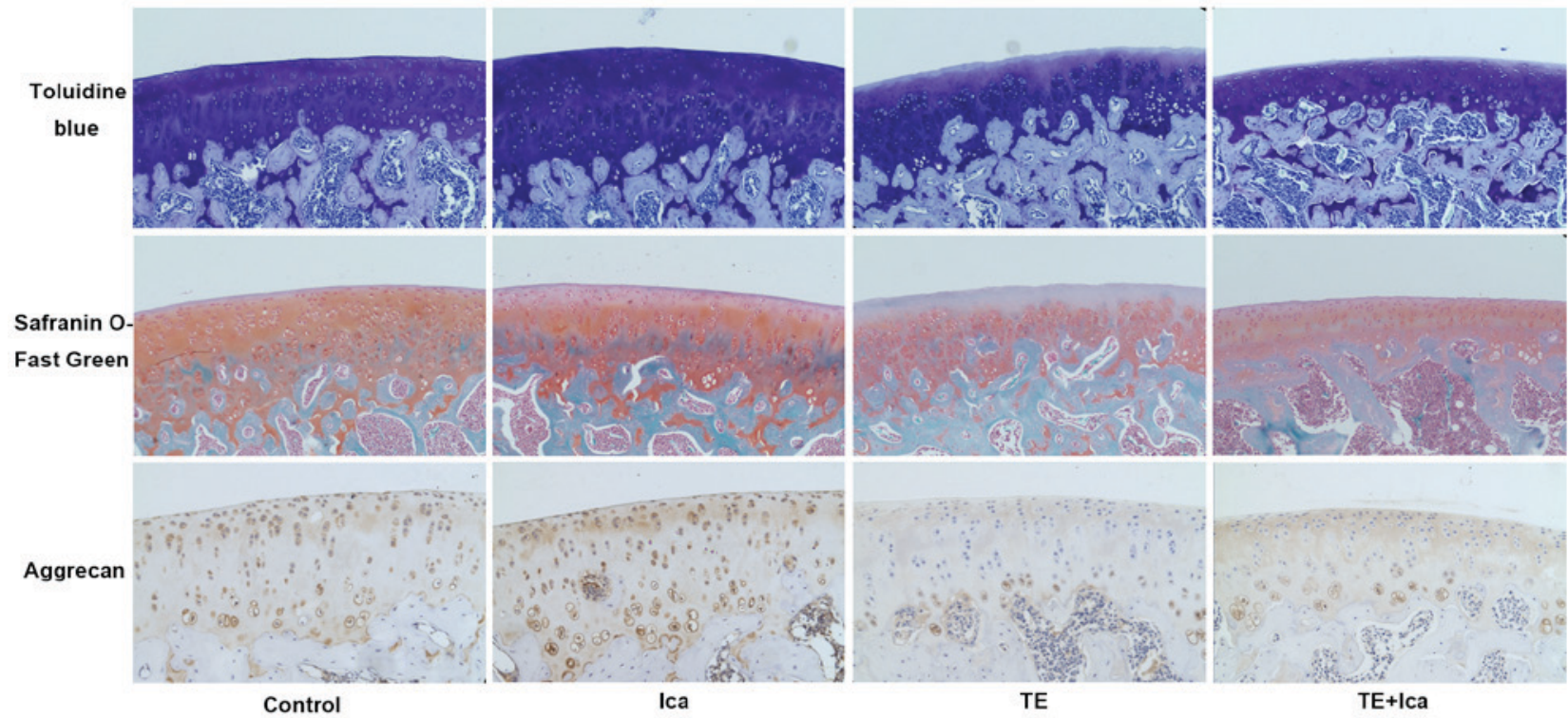

B

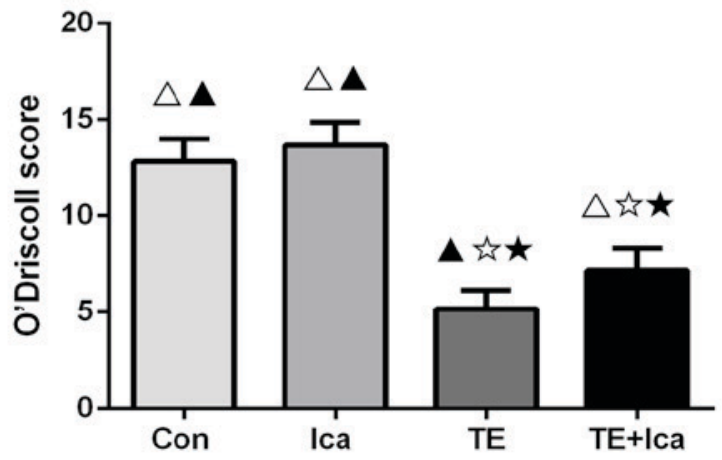

Figure 4. Icariin attenuates cartilage degeneration in vivo. Full-thickness cartilage defects were created in rats, and early treadmill running was used to accelerate the progression of post-traumatic osteoarthritis. (A) Toluidine blue and Safranin O-Fast Green staining revealed cartilage thicknesses and the proteoglycan levels (magnification, x100). Immunochemical staining was used to detect aggrecan, the major component of the cartilage extracellular matrix (magnification, x200). (B) The O'Driscoll scores of different groups are shown $\left({ }^{\mathrm{A}} \mathrm{P}<0.05\right.$ compared with the TE group; ${ }^{\star} \mathrm{P}<0.05$ compared with the TE+Ica group; ${ }^{\star} \mathrm{P}<0.05$ compared with the Con group; ${ }^{\star} \mathrm{P}<0.05$ compared with the Ica group). Con, control; Ica, icariin; TE, treadmill exercise.

was clearly expressed in the icariin-treated group, but the treadmill group exhibited reduced expression in the upper and middle cartilage layers; addition of icariin partly reversed this decline (Fig. 4A). When PTOA severity was assessed by the O'Driscoll system (19), the scores of the control (12.833 \pm 1.169$)$, icariin (13.667 \pm 1.211$)$, and treadmill + icariin $(6.833 \pm 1.472)$ groups were all significantly higher compared that of the treadmill group (5.167 \pm 0.983$)$ (Fig. 4B). Therefore, icariin was shown to improve the histological cartilage phenotype.

ERK phosphorylation is involved in icariin-mediated IFT88 expression. siRNA was used to knock down IFT88 gene expression, and this revealed that icariin rescued the reductions in COL-II and SOX9 expression levels induced by siRNA (Fig. 5A). siRNA downregulated the expression of endogenous IFT88 and phosphorylated ERK (Fig. 5B). Icariin (10 $\mu \mathrm{mol} / \mathrm{l})$ promoted IFT88 expression and ERK phosphorylation compared with the levels noted in the presence of IFT88 siRNA (Fig. 5C). Inhibition of ERK phosphorylation by PD0325901 clearly reduced IFT88 expression; icariin did not rescue this decline (Fig. 5D). Therefore, ERK phosphorylation may be involved in icariin-mediated IFT88 expression during maintenance of the chondrocyte phenotype.

\section{Discussion}

Maintenance of the cartilage phenotype is a major concern during cartilage tissue engineering. Although various biological materials and recombinant cytokines may be of assistance, these materials degrade rapidly, exhibit unpredictable side-effects, and are very costly (24-26). In the present study, a Herba Epimedii extract, icariin, was shown to aid in maintenance of the cartilage phenotype, and the IFT88 protein of primary cilia served an important role in this context. Icariin exerts multiple functions. Li et al (7) demonstrated that, in animal models, icariin-impregnated hydrogel scaffolds efficiently promoted the repair of defects in superficial cartilage and osteochondral regions (7). Zhang et al (27) showed that icariin promoted chondrocyte clustering and ECM secretion (27). In the present study, icariin was shown to promote 
A
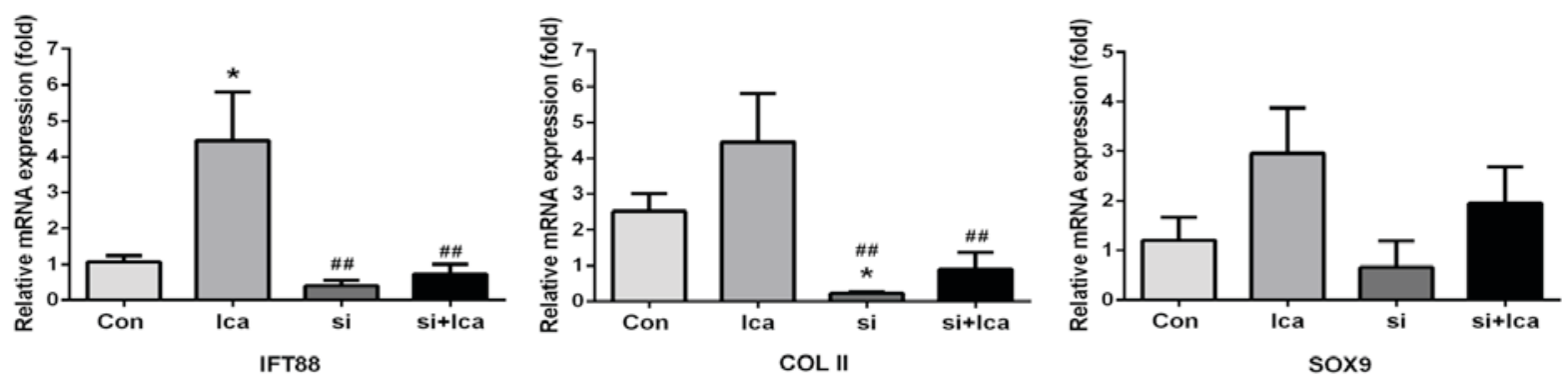

B
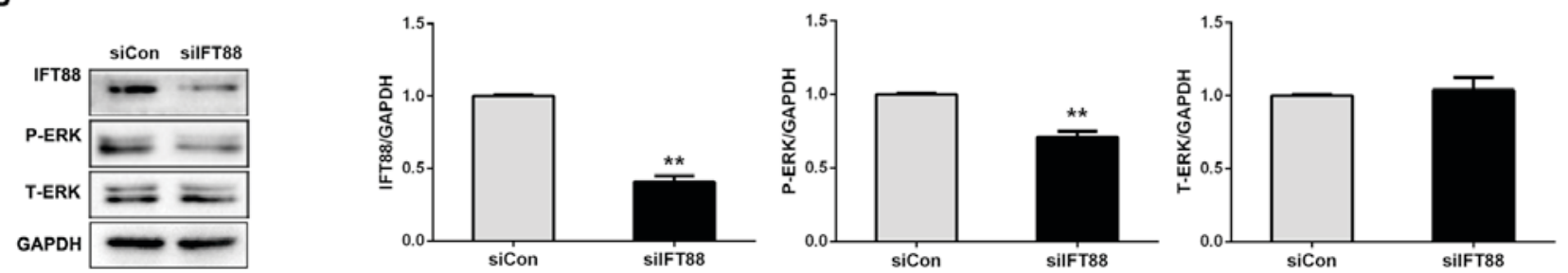

C
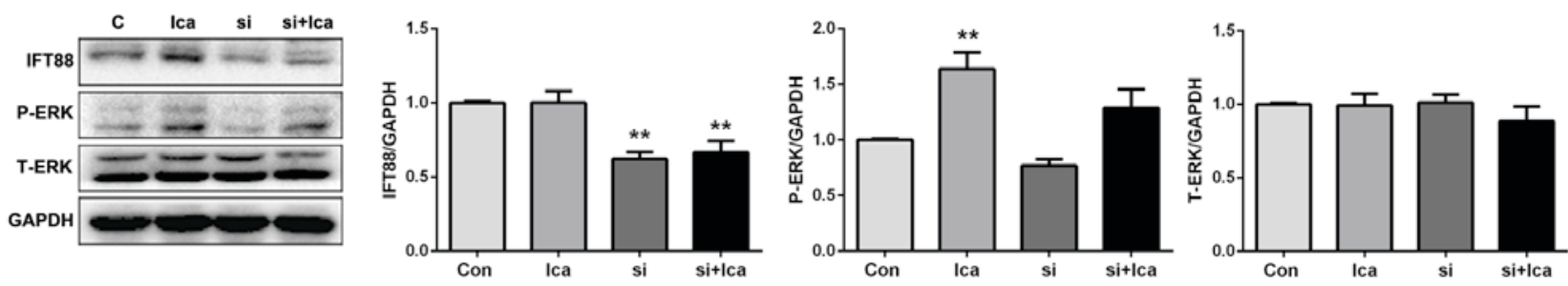

D
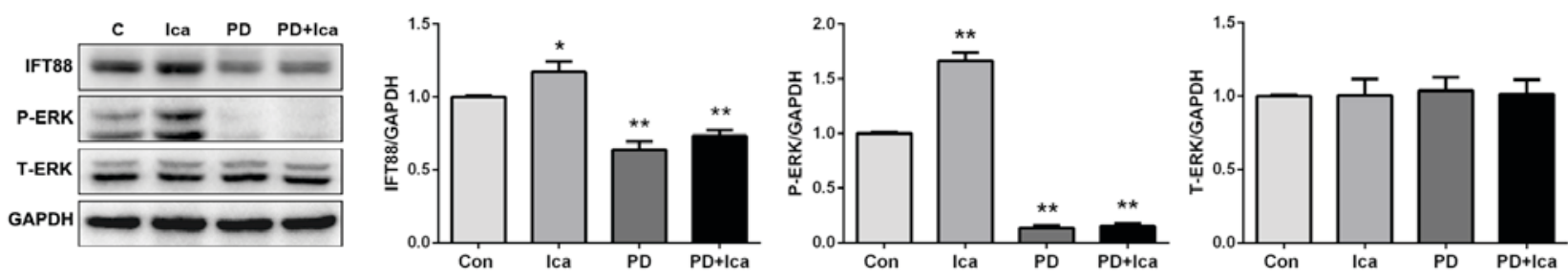

Figure 5. Icariin regulates IFT88 expression via the ERK signalling pathway. (A) Quantitative polymerase chain reaction measuring the expression levels of the IFT88 and various phenotype-associated genes after transformation of IFT88 siRNA ( ${ }^{*} \mathrm{P}<0.05$ compared with the control group; ${ }^{* \#} \mathrm{P}<0.01$ compared with the icariin group). (B) IFT88 knockdown reduced the expression of phosphorylated ERK (" $\mathrm{P}<0.01$ compared with the siCon group). (C) Icariin promoted expression of IFT88 and phosphorylated ERK, and slightly increased ERK phosphorylation, after transformation of IFT88 siRNA ("P<0.01 compared with the control group). (D) An ERK inhibitor reduced IFT88 expression. Icariin upregulated IFT88 expression, but could not restore such expression in the presence of the ERK inhibitor, PD0325901 ( $\mathrm{P}<0.05 ;{ }^{* *} \mathrm{P}<0.01$ compared with the control group). ERK, extracellular signal-regulated kinase; IFT88, intraflagellar transport protein 88; Ica, icariin; PD, PD0325901, Con, control; T-ERK, total ERK; P-ERK, phosphorylated ERK.

cellular proliferation, and expression of the cartilage phenotype genes, COL-II and SOX9, in progenitor chondrocytic cells and primary chondrocytes was enhanced. These findings suggested that icariin may be valuable in cartilage tissue engineering. However, the mechanism by which icariin promotes cartilage repair has yet to be fully elucidated.

IFT88, an intraflagellar transport protein, carries cargos into or out of primary cilia (9). This protein has been shown to be very important in the development of cartilage $(10,28)$. IFT88 affects actin organisation within chondrocytes, and also the biomechanical properties of cartilage (28). IFT88 mutations render cartilage 'osteoarthritis-like', with reduction of the compressive modulus (29). IFT88 fulfils a major role in the columnar organisation of growth plate cartilage (15). In the present study, IFT88 was shown to be widely distributed in the cytoplasm, although it was principally concentrated along the ciliary axoneme. Icariin promoted ciliary assembly, but did not affect ciliary length. Icariin upregulated IFT88 expression at both the gene and the protein level, promoted ECM secretion, and increased the expression of COL-II and SOX9. Therefore, icariin enhanced maintenance of the cartilage phenotype and IFT88 exerted an important role in icariin-mediated ECM secretion. IFT88 knockdown suppressed ERK phosphorylation, and icariin partially rescued this reduction. Thus, there may be feedback between the IFT88 expression level and ERK phosphorylation in operation, and icariin contributes positively to such regulation. It was therefore confirmed that icariin increased ciliary assembly, enhanced IFT88 expression, promoted ciliary transportation, stimulated ERK phosphorylation, and induced SOX9 and COL-II gene expression to promote cartilage matrix secretion. Primary cilia and the IFT88 protein may serve important roles during cartilage 
development. However, the gene expression profile and the signaling systems involved require further study.

In conclusion, the present study explored the role exerted by the ciliary protein IFT88 in icariin-mediated maintenance of cartilage phenotype in progenitor cells and in primary chondrocytes. Icariin promoted ciliary assembly, enhanced intraflagellar transportation, induced ERK phosphorylation, and stimulated cartilage matrix secretion. Icariin thus aids in the maintenance of cartilage phenotype, and the present study has provided the theoretical basis for the use of icariin in cartilage tissue engineering.

\section{Acknowledgements}

This study was supported by the National Natural Science Foundation of China (grant nos. 81572094 and 81371915).

\section{References}

1. Varady NH and Grodzinsky AJ: Osteoarthritis year in review 2015: Mechanics. Osteoarthritis Cartilage 24: 27-35, 2016.

2. Jiang Z, Hu B, Wang J, Tang Q, Tan Y, Xiang J and Liu J: Effect of icariin on cyclic GMP levels and on the mRNA expression of cGMP-binding cGMP-specific phosphodiesterase (PDE5) in penile cavernosum. J Huazhong Univ Sci Technolog Med Sci 26: 460-462, 2006.

3. Schluesener JK and Schluesener H: Plant polyphenols in the treatment of age-associated diseases: Revealing the pleiotropic effects of icariin by network analysis. Mol Nutr Food Res 58: 49-60, 2014.

4. Song YH, Li BS, Chen XM and Cai H: Ethanol extract from Epimedium brevicornum attenuates left ventricular dysfunction and cardiac remodeling through down-regulating matrix metalloproteinase-2 and -9 activity and myocardial apoptosis in rats with congestive heart failure. Int J Mol Med 21: 117-124, 2008.

5. Wu Y, Xia L, Zhou Y, Xu Y and Jiang X: Icariin induces osteogenic differentiation of bone mesenchymal stem cells in a MAPK-dependent manner. Cell Prolif 48: 375-384, 2015

6. Zhai YK, Guo XY, Ge BF, Zhen P, Ma XN, Zhou J, Ma HP, Xian CJ and Chen KM: Icariin stimulates the osteogenic differentiation of rat bone marrow stromal cells via activating the PI3K-AKT-eNOS-NO-cGMP-PKG. Bone 66: 189-198, 2014.

7. Li D, Yuan T, Zhang X, Xiao Y, Wang R, Fan Y and Zhang X: Icariin: A potential promoting compound for cartilage tissue engineering. Osteoarthritis Cartilage 20: 1647-1656, 2012.

8. Muhammad H, Rais Y, Miosge N and Ornan EM: The primary cilium as a dual sensor of mechanochemical signals in chondrocytes. Cell Mol Life Sci 69: 2101-2107, 2012.

9. Scholey JM: Intraflagellar transport. Annu Rev Cell Dev Biol 19: 423-443, 2003

10. Haycraft CJ, Zhang Q, Song B, Jackson WS, Detloff PJ, Serra R and Yoder BK: Intraflagellar transport is essential for endochondral bone formation. Development 134: 307-316, 2007.

11. Thompson CL, Chapple JP and Knight MM: Primary cilia disassembly down-regulates mechanosensitive hedgehog signalling: A feedback mechanism controlling ADAMTS-5 expression in chondrocytes. Osteoarthritis Cartilage 22: 490-498, 2014.

12. Shao YY, Wang L, Welter JF and Ballock RT: Primary cilia modulate Ihh signal transduction in response to hydrostatic loading of growth plate chondrocytes. Bone 50: 79-84, 2012.
13. Hoey DA, Tormey S, Ramcharan S, O'Brien FJ and Jacobs CR: Primary cilia-mediated mechanotransduction in human mesenchymal stem cells. Stem Cells 30: 2561-2570, 2012.

14. Poole CA, Jensen CG, Snyder JA, Gray CG, Hermanutz VL and Wheatley DN: Confocal analysis of primary cilia structure and colocalization with the Golgi apparatus in chondrocytes and aortic smooth muscle cells. Cell Biol Int 21: 483-494, 1997.

15. Song B, Haycraft CJ, Seo HS, Yoder BK and Serra R: Development of the post-natal growth plate requires intraflagellar transport proteins. Dev Biol 305: 202-216, 2007.

16. Ho L, Ali SA, Al-Jazrawe M, Kandel R, Wunder JS and Alman BA: Primary cilia attenuate hedgehog signalling in neoplastic chondrocytes. Oncogene 32: 5388-5396, 2013.

17. Lu W, Shi J, Zhang J, Lv Z, Guo F, Huang H, Zhu W and Chen A: CXCL12/CXCR4 Axis regulates aggrecanase activation and cartilage degradation in a post-traumatic osteoarthritis rat model. Int J Mol Sci 17: pii: E1522, 2016.

18. O'Driscoll SW, Keeley FW and Salter RB: Durability of regenerated articular cartilage produced by free autogenous periosteal grafts in major full-thickness defects in joint surfaces under the influence of continuous passive motion. A follow-up report at one year. J Bone Joint Surg Am 70: 595-606, 1988.

19. Song JQ, Dong F, Li X, Xu CP, Cui Z, Jiang N, Jia JJ and Yu B: Effect of treadmill exercise timing on repair of full-thickness defects of articular cartilage by bone-derived mesenchymal stem cells: An experimental investigation in rats. PLoS One 9: e90858, 2014.

20. Nian H, Ma MH, Nian SS and Xu LL: Antiosteoporotic activity of icariin in ovariectomized rats. Phytomedicine 16: 320-326, 2009.

21. de Andrea CE, Zhu JF, Jin H, Bovée JV and Jones KB: Cell cycle deregulation and mosaic loss of Ext1 drive peripheral chondrosarcomagenesis in the mouse and reveal an intrinsic cilia deficiency. J Pathol 236: 210-218, 2015.

22. Ke YN and Yang WX: Primary cilium: An elaborate structure that blocks cell division? Gene 547: 175-185, 2014.

23. Gibson BG and Briggs MD: The aggrecanopathies; an evolving phenotypic spectrum of human genetic skeletal diseases. Orphanet J Rare Dis 11: 86, 2016.

24. Hunziker EB, Lippuner K, Keel MJ and Shintani N: An educational review of cartilage repair: Precepts \& practice - myths \& misconceptions-progress \& prospects. Osteoarthritis Cartilage 23: 334-350, 2015.

25. Demoor M, Ollitrault D, Gomez-Leduc T, Bouyoucef M, Hervieu M, Fabre H, Lafont J, Denoix JM, Audigié F, Mallein-Gerin F, et al: Cartilage tissue engineering: Molecular control of chondrocyte differentiation for proper cartilage matrix reconstruction. Biochim Biophys Acta 1840: 2414-2440, 2014.

26. Johnstone B, Alini M, Cucchiarini M, Dodge GR, Eglin D, Guilak F, Madry H, Mata A, Mauck RL, Semino CE and Stoddart MJ: Tissue engineering for articular cartilage repair-the state of the art. Eur Cell Mater 25: 248-267, 2013.

27. Zhang L, Zhang X, Li KF, Li DX, Xiao YM, Fan YJ and Zhang XD: Icariin promotes extracellular matrix synthesis and gene expression of chondrocytes in vitro. Phytother Res 26: 1385-1392, 2012.

28. Wang Z, Wann AK, Thompson CL, Hassen A, Wang W and Knight MM: IFT88 influences chondrocyte actin organization and biomechanics. Osteoarthritis Cartilage 24: 544-554, 2016.

29. Irianto J, Ramaswamy G, Serra R and Knight MM: Depletion of chondrocyte primary cilia reduces the compressive modulus of articular cartilage. J Biomech 47: 579-582, 2014.

(i) $\Theta$ This work is licensed under a Creative Commons Attribution-NonCommercial-NoDerivatives 4.0 International (CC BY-NC-ND 4.0) License. 